\title{
A Case Report on Rare Disease - Pemphigus Foliaceus
}

\author{
Manohar Reddy YV $^{1 *}$, Reddy Mahesh $N^{1}$, Sandeep P ${ }^{1}$, Divya G ${ }^{2}$ \\ ${ }^{1}$ Pharm D, Intern, Sri Padmavathi School of Pharmacy, Tiruchanoor, Tirupathi, India \\ ${ }^{2}$ Assistant professor, Sri Padmavathi School of Pharmacy, Tiruchanoor, Tirupathi, India
}

Submission: January 29, 2020; Published: February 13, 2020

*Corresponding author: Manohar Reddy YV, Pharm D, Intern, Sri Padmavathi School of Pharmacy, Tiruchanoor, Tirupathi, India

Abstract

Auto immune bullous diseases are very rare diseases of the skin out of which Pemphigus foliaceus an autoimmune bullous disease affecting mainly the skin but not to the mucous membrane. Occurrence is $0.5-3.2$ cases in one lakh population which is very less when compared to other forms of bullous diseases. A 48 years old male patient working as cooly admitted in the dermatology ward with complaints of reddish crusted and lesions over the body. He was prescribed with antibiotics along with Dexamethasone. On sudden stoppage of dexamethasone patient experienced shaking legs and hands, later the symptoms of the patient decreased by tampering the dose of corticosteroid. In some population altered Human Leucocyte Antigen causes the production of autoantibodies against the desmosomal proteins which on further causes the development of disease. Sudden shifting of one interleukins from one type to another may results in recurrence of the disease. Generally some of the drugs, over exposure to sunlight and certain stimulants lead to the development of disease. Pemphigus foliaceus is not a life threatening disease certain life style changes may alter the disease state.

Keywords: Pemphigus foliaceus, Human Leucocyte Antigen, Desmosomal proteins, Corticosteroids, Life style changes

\section{Introduction}

Blistering skin disorders can be divided into immunobullous diseases, blistering skin infections and others. Pemphigus comes under the category of immunobullous diseases [1]. The term Pemphigus, was derived from the Greek word Pemphix which means bubble or blister, was originally used by Wichman in 1791, for describing a chronic blistering disease [2]. Pemphigus is a group of rare diseases that causes blisters. Pemphigus has an incidence of 0.5-3.2 cases in 1 lakh population. There are different types of pemphigus which are Pemphigus vulgaris, Pemphigus foliaceus, Drug-induced pemphigus, Fogo selvage, Paraneoplastic pemphigus (PNP), IgA pemphigus. Among different types of pemphigus, pemphigus vulgaris is the most common one [3]. Pemphigus foliaceus is an autoimmune blistering disease of the skin but not to the mucous membrane. In this particular disease the own body synthesises auto antibodies against own proteins in body. In most cases, the disease develops on its own, but certain medications also can cause pemphigus to develop. Pemphigus commonly affects people aged between 40 and 60 years. The disease can last for many years [4]. Generally pemphigus foliaceus is managed with corticosteroids, emmolients, creams and supportive therapy.

\section{Case Details}

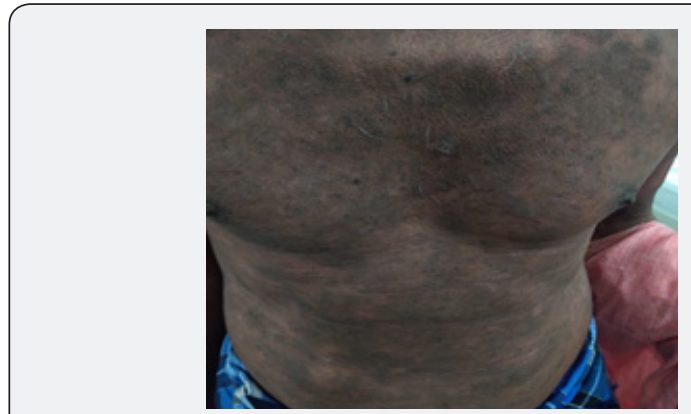

Figure 1: Blisters on the chest.

A 48 years old male patient admitted in dermatology ward working as cooly with a chief complaints of crusted areas with reddish in appearance and some lesions over the body since one month. His present illness started as fluid filled lesions developed over the trunk (Figure 1) and they gradually spread to scalp , fore head (Figure 2), back midline region (Figure 3), face , retroau- 
ricular area and legs region. The blisters burst on its own to form corn flake crasts over the body with in one day. H/O of similar complaints in the past and took treatment from government hospital. Not a known case of any chronic diseases and not an alcoholic and smoker. All his laboratory findings seems to be normal on all days except the dermatological findings. Initially the prescriber prescribes : T. Amoxyclav 625mg BD, T. Pantop 40mg BD, T. Cetirizine $10 \mathrm{mg} \mathrm{H} / \mathrm{S}$, T. Paracetamol $500 \mathrm{mg}$ BD, T.B complex/T.VIT C OD, Potassium permanganate soaks and Fusidic acid ointment for F/E/A. On the next day same therapy is continued but potassium permanganate soaks is changed to Saline soaks. Same therapy is continued but on 5th day Inj. Dexamethasone 2cc IM OD started and Fusidic acid ointment is stopped on the same day. Betamethasone ointment is added on 11th day of treatment and stopped saline soaks. On 16th day of treatment Emollient is added. During the course of treatment dexamethasone injection is withdrawed on 18th day and due to this patient experienced shaking legs and hands and on the next day 0.5 cc Dexamethasone administered to patient. On the final 20th day all his complaints alleviated and patient discharged.

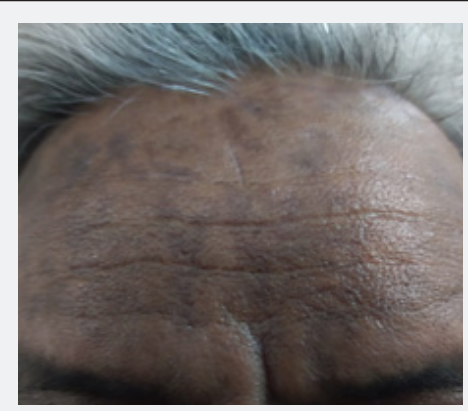

Figure 2: Corn flake like marks over the forehead.

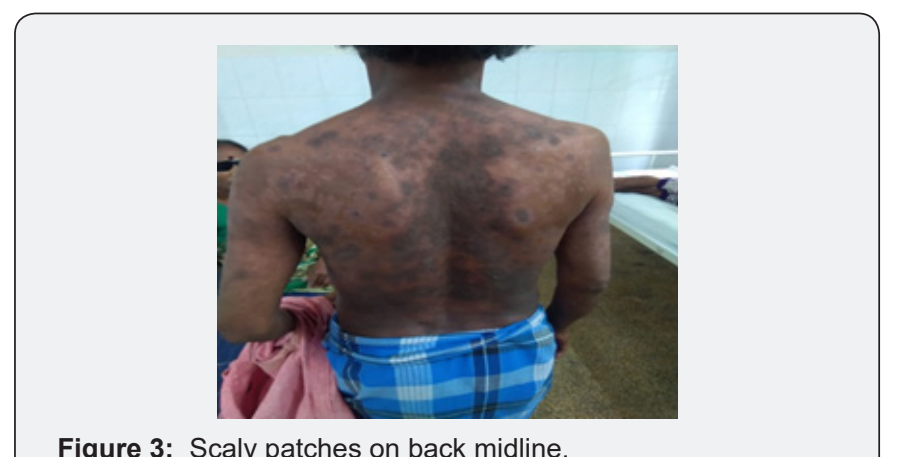

Figure 3: Scaly patches on back midline.

\section{Discussion}

Pemphigus foliaceus is an autoimmune disease which is marked by the presence of autoantibodies against the cell surface of keratinocytes as a result it causes the destruction of epidermal cell junctions. This group of autoimmune diseases can causes blisters on the upper parts of the epidermis, either in the granular layer or just beneath the stratum corneum [5]. The underlined pathogenesis in all forms of pemphigus involves the development of autoantibodies to the desmosomal proteins, as they found in many areas of the body, which play a major role in the epidermal layers of the integumentary system [6]. The disease usually occurs in patients with certain Human Leucocyte Antigen (HLA) genotypes causing the generation of B-cells responsible for the specific autoantibodies. The activation of these B-cells requires a complex interaction with CD4+ T helper 2 (Th2) cells and it is this Th2 cell over-activation that leads to the autoantibody production a hallmark feature in Pemphigus foliaceus. Th2 cells are known for secreting multiple interleukins (IL), of which IL-4 plays a major role in pemphigus and the humoral immune response [7].

IL-4 promotes antibody production by primed B cells and an important isotype that switches from IgG1 to IgG4 antibodies which have been shown to be important in the active form of PF. IL-4 also perpetuates the disease by causing naive CD4+ T cells to differentiate into Th2 cells. The production of autoantibodies and epitope binding is promoted to lose the adhesions between desmosomes leading to separation of keratinocytes which is directly related to disease activity. When pemphigus is sawn as a remission there is a known up-regulation of IL-10 and a T helper 1 response that induces antibody isotype switching from IgG4 back to IgG1 [8].

As a result this PF can leads to Small fluid-filled skin blisters that typically begin on the face, scalp, or trunk. Ruptured blisters that cause sores, pockets, or scars in the skin. Scaly, inflamed, painful patches on the skin. These patches occur after blisters burst. Burning, pain, and itching at the site of the blisters. Chronic skin infections due to ruptured and irritated blisters. Generally pemphigus foliaceous is caused by ethnicity and geographical areas such as india, gender, sun exposure and even some medications may also causes PF. Skin biopsy and general physical examination may confirms PF. PF is not an life threatening disease. This can be managed by Avoidance of risk factor, Corticosteroids, anti-inflammatory agents, antibiotics and at certain extreme conditions immune suppressants may also use [9]. Corticosteroid such as Dexamethasone in this patient decreases the immune response and promotes the healing of blisters.

\section{Conclusion}

Pemphigus foliaceus is a benign disease of long duration that responds to treatment with the corticosteroids and from which the patient may eventually recover. At times, no treatment is required for the management of patients with relatively few lesions. Systemic steroids suppress the cutaneous flares and improve general well-being, but long-term steroid therapy usually is not indicated.

\section{References}

1. Nicloe yi zhen chaing (2014) A handbook for medical students and junior doctors. $2^{\text {nd }}$ Ed $^{\text {n }}$ British Association of Dermatologist, 65.

2. Lever W (1953) Pemphigus. Medicine (Baltimore) 32(1): 1-123.

3. Vanessa Ngan (2003) Staff Write, Pemphigus foliaceus. 
4. Marcel F Jonkman (2014) JAMA Dermatology Patient Page. Pemphigus 150(6): 680 .

5. Ghalamkarpour F, Azin A, Somayeh H (2012) Pemphigus Foliaceus, indian pediatrics 49: 240-241.

6. Christopher D (2016) Pemphigus: Pathogenesis to Treatment, advances in autoimmune diseases, rhodeisland medical journal: 28-30.
7. Giordano CN, Sinha AA (2012) Cytokine networks in Pemphigus vulgaris, an integrated viewpoint. Autoimmunity 45(6): 427-439.

8. Di Zenzo G, Amber KT, Sayar BS, Müller EJ, Borradori L (2016) Immune response in pemphigus and beyond progresses and emerging concepts, Semin Immunopathol 38(1): 57-74.

9. Suzanne Falck, Zawn Villines (2017) Pemphigus foliaceus: What's to know.

\section{Your next submission with Juniper Publishers will reach you the below assets}

Commons Attribution 4.0 License

DOI: 10.19080/JOJDC.2020.02.555584 